\title{
Labeling effects on memory for nonsense pictures
}

\author{
ROBERTA L. KLATZKY and KAREN RAFNEL \\ University of California, Santa Barbara, California 98106
}

\begin{abstract}
It has been shown that labeling nonsense pictures improves memory for them, but how the label takes effect is uncertain. In this study subjects viewed nonsense pictures with or without labels; in the former case, the labels were either meaningful or nonmeaningful with respect to the pictures. Then the subjects took part in a free recall test, followed by recall in the presence of cues. Only the meaningful labels facilitated free recall of the pictures, but both types of labels facilitated cued recall. The results suggest that a meaningful label affects picture encoding by providing a conceptual interpretation for the picture; a nonmeaningful label, in contrast, appears to provide only an ad hoc associative cue.
\end{abstract}

When people are shown pictures to remember, is their recall facilitated when they are able to interpret the pictures with respect to some conceptual schema? An affirmative answer was provided for this question by Bower, Karlin, and Dueck (1975). They presented nonsense pictures, called "droodles" (Price, 1972, 1973) to two groups of subjects. One group was shown a label along with each droodle; it had the effect of rendering the droodle sensible. The other group saw the droodles without labels. The group given labels performed better on a subsequent free recall (reproduction) test of the droodles. Bower et al. interpreted these results as indicating that the meaningful interpretation of an otherwise nonsensical picture (as is the case when it is paired with a meaningful label) facilitates its memorial representation and subsequent retrieval. A similar effect has been found for verbal material (e.g., Bransford \& Johnson, 1972; Dooling \& Lachman, 1971).

However, the Bower et al. (1975) result is subject to alternative interpretation because their experiment compared only two groups: subjects given meaningful labels and those given no labels at all. The possibility remains that the observed effect of labels was not due to their meaningfulness, but to some property of labels per se. It has been demonstrated that labeling, even with arbitrary labels, has a facilitative effect on pictorial memory. For example, Santa and his colleagues (e.g., Nicosia \& Santa, 1975; Santa \& Ranken, 1972) have found that even arbitrary or nonrepresentative verbal labels aid reproduction and redintegration of pictorial stimuii. Santa et al. have suggested that verbal labeling may improve memory for forms by providing subjects with an ad hoc scheme for interitem organization; this contrasts with the conceptual-interpretation hypothesis of Bower et al. Another possible explanation for the Bower et al. results is that giving subjects labels may heighten their motivation to study the droodles, independently of any meaning added by the labels.

This research was supported by Grant MH 25090 from the National Institute of Mental Health to the first author.
The present experiment was intended to assess whether or not the effect of labeling on recall of nonsense pictures depends on the meanings the labels provide. To this end, two groups of subjects were presented with droodles: one group with labels (the label group) and the other without labels (the no-label group). Further, in the label group, each label was either meaningfully representative or nonmeaningful with respect to the droodle with which it was paired. Later, each subject was given a reproduction test. If labels facilitate recall of nonsense forms by making them interpretable, reproduction of forms given nonmeaningful labels should be no better than that of forms given no labels at all. On the other hand, a positive effect of nonmeaningful labels would suggest that the nature of the labeling effect is something other than to provide the picture with an immediate conceptual organization.

In addition to questioning whether the effect of labeling relies on the label's being meaningful, one should also consider the locus of the effect. Freedman and Haber (1974) and Wiseman and Neisser (Note 1) have reported findings from experiments related to the present one which suggest that the locus might be at the encoding stage. In those studies, subjects' ability to recognize previously seen Mooney figures (which are difficult to interpret) depended on their having arrived at an interpretation of the figures at the time they were presented.

In the present study, a cued recall test was given subsequent to the initial free recall test, and the two were compared to investigate the locus of the labeling effect. The change in performance from free to cued recall can be used to determine if there is a facilitative effect of providing cues at the time of retrieval. This effect of retrieval cues can be assessed both for subjects who had similar cues at the time of encoding (the label group) and for those who did not (the no-label group). If labeling can take effect at the time of retrieval alone, substantial improvement over the two tests should be observed for the no-label group. If an effect of labeling occurs at the time of encoding, the label group 
should be superior to the no-label group on free recall, and should even improve their relative performance at cued recall, when the cues are reinstated. The relative improvement across tasks for the droodles given meaningful and nonmeaningful labels can then be compared.

\section{METHOD}

Subjects

The subjects were 36 undergraduates at the University of California, Santa Barbara. Their participation fulfilled partial requirements for an introductory course in psychology.

\section{Stimuli}

The stimuli consisted of a pool of 45 droodles, chosen from booklets by Price $(1972,1973)$. Each droodle was a simple black line drawing which is generally uninterpretable unless its viewer is supplied with a verbal cue indicating what the drawing represents. The droodles were photographed and made into slides, with each droodle brought into a standardized size. The stimuli also included the name for each droodle as supplied by Price. A few of these labels were modified, such that those containing more than six words were shortened to within that length (this modification did not affect the gist of the label).

That each label was meaningful relative to its corresponding (according to Price) droodle, but not relative to some other droodle with which it was arbitrarily paired, was determined by a set of six graduate-student raters. Each rater was given a set of droodles and a set of either the corresponding labels or some arbitrarily designated labels and was told to try to match each droodle with a label. The task was performed with essentially $100 \%$ accuracy with the meaningful labels and at only $2 \%$ accuracy with the arbitrarily designated labels.

\section{Design}

The 36 undergraduates were randomly assigned to one of two groups of 18 subjects each. Each subject was shown 30 droodles. One group (the label group) saw a label along with each droodle. Within this group, half the droodles were paired with their corresponding meaningful labels, forming a set of meaningful (M) droodle-label pairs. The other half of the droodles were arbitrarily paired with labels that actually corresponded to some other droodle not seen by the subject; this formed a nonmeaningful (NM) set of droodle-label pairs. The second group of subjects (the no-label group) initially saw just 30 droodles, without labels. However, half of these droodles were designated as members of a meaningful (M) set and half as members of a nonmeaningful (NM) set, according to the type of label that would subsequently be provided for each of them in cued recall testing. (The use of droodles and labels was counterbalanced across subjects so that each droodle and each label occurred in all possible conditions.)

Following initial presentation of droodles with or without labels, each subject took part in a free recall test in which he drew all the droodles he could remember. After the free recall test, each subject saw a list of 30 labels and was instructed to draw any droodles the labels brought to mind; this provided a cued recall test.

In summary, the experimental conditions included two groups of subjects-having labels or no labels at the time the droodles were presented-and within each group, two kinds of droodles-those paired with meaningful labels (M) and those paired with nonmeaningful labels (NM). Recall in these conditions was measured with both a free recall and a subsequent cued recall test.

\section{Procedure}

The subjects were tested in groups of three; they sat at adjacent tables in a common room. The droodles were presented at a rate of one every $10 \mathrm{sec}$ and were randomized with respect to the meaningfulness factor. If labels were presented at this time, each was projected just beneath the corresponding droodle and simultaneously with it. Immediately following the completed presentation of the 30 droodles, the subjects were given $8 \mathrm{~min}$ to draw as many as they could remember, in any order. Picture reproductions were drawn on $8 \frac{1}{2} \times 11$ in sheets that had been marked off into 3 by 3 matrices. A subject drew a picture he could recall into one of the nine squares on a sheet and was free to use as many sheets as he needed to complete the work. Subjects were instructed that they should not attempt to draw in great detail, but should try to convey the gist of each picture. Next, subjects in the label group were given $6 \mathrm{~min}$ to write any labels they could recall under the corresponding droodle; those in the no-label group had equivalent time to reinspect their droodles.

Following the free recall task, subjects took part in the test of cued recall. For this test, sheets similar to the free recall test sheets were used, except that a label appeared in each of 30 squares. For subjects in the label condition, the labels which appeared in the squares were precisely those seen during the initial presentation, but in a randomized presentation order. Subjects in the no-label group, however, were seeing labels for the first time. For this group, half of the labels appearing in the squares corresponded to droodles they had previously seen, forming meaningful droodle-label pairs. The remaining half of the labels, however, were not meaningful with respect to any previously seen droodles. Instead, for purposes of scoring the cued recall, each nonmeaningful label had been paired arbitrarily with one of the remaining droodles. In both labeling conditions, subjects were directed to draw in any pictures which seemed "to go" with the labels supplied; they were given $8 \mathrm{~min}$ to finish their drawings. Upon completion of the cued recall task, the subjects were debriefed and dismissed.

\section{RESULTS}

The data were mean number of droodles correctly reproduced (out of a possible 15) in each condition. The criteria for considering a reproduction correct were lenient, requiring only that it generally resemble the features of the original and that the same droodle was not drawn twice. Reproductions were judged by three independent graduate-student raters (not the same as those who originally rated the stimuli), and concurrence of two out of three raters was used as the criterion for scoring a droodle correct or incorrect. (As reported by Bower et al., 1975, the scoring task was quite easy, with high rater agreement.)

Data for each group of subjects (label and no-label), meaningful (M) and nonmeaningful (NM) droodles, and free and cued recall tests are presented in Table 1." These data were submitted to a three-way analysis of variance, with factors (1) Labeling condition at presentation, (2) Meaningfulness of label, and (3) type of Test, with repeated measures on the last two factors. The analysis revealed significant main effects of Labeling $[F(1,34)=99.23, \quad p<.001, \quad$ MSe $=10.08] \quad$ and Meaningfulness $[F(1,34)=69.19, p<.001, \mathrm{MSe}=3.7]$, but not type of Test $[\mathrm{F}(1,34)=2.64, \mathrm{p}<.25$, $\mathrm{MSe}=3.04]$. Significant interactions were found for Labeling by Test Type $[\mathrm{F}(1,34)=286.27, \mathrm{p}<.001$, MSe $=3.04]$, Meaningfulness by Test Type 
Table 1

Mean Number of Correct Reproductions for Free and Cued Recall for Each Labeling Condition and Label Type

\begin{tabular}{lcc}
\hline & \multicolumn{2}{c}{ Labeling Condition } \\
\cline { 3 - 3 } & Label & No Label \\
\hline Free Recall & & \\
$\quad$ Meaningful Label & 8.4 & 6.8 \\
$\quad$ Nonmeaningful Label & 5.8 & 6.8 \\
$\quad$ Cued Recall & & \\
$\quad$ Meaningful Label & 14.4 & 4.6 \\
$\quad$ Nonmeaningful Label & 10.6 & .2 \\
\hline
\end{tabular}

$[F(1,34)=69.44, p<.001, \mathrm{MSe}=2.61]$, and Labeling by Meaningfulness by Test Type $[\mathrm{F}(1,34)=24.98$, $\mathrm{p}<.005, \mathrm{MSe}=2.61]$.

To assess the principal questions of the study, planned comparisons were then conducted. (These comparisons all use the three-way interaction term as the error mean square; $\mathrm{MSe}=2.61$.) A significant difference between the label and no-label groups was found for free recall of $M$ droodles $[F(1,34)=9.58$, $p<.01]$. This finding replicates that of Bower et al. (1975). However, the corresponding difference between the label and no-label groups for NM droodles was not significant $[F(1,34)=3.44, p>.05]$. Thus, free recall was not significantly better for droodles paired with a nonmeaningful label than for those given no label at all. Within the label group, free recall for $M$ droodles was found to be significantly higher than for NM droodles $[F(1,34)=25.5, p<.001]$. Taken in conjunction, the three comparisons reveal that, relative to no labels, meaningful labels facilitate free recall of droodles but nonmeaningful labels do not.

The two-way interaction between the Labeling and Test-Type factors was assessed within each of the $M$ and NM conditions, and both were significant $[F(1,34)=114.99, \quad p<.001$, and $F(1,34)=228.01$, $\mathrm{p}<.001$, for the $M$ and $N M$ conditions, respectively]. Thus, within both the $M$ and NM conditions, performance improves from free to cued recall for the group given labels at presentation, but not for those without labels at presentation.

\section{DISCUSSION}

The principal question addressed by the present study was whether the beneficial effect on free recall of labeling a nonsense picture was dependent upon the given label's being meaningful with respect to the picture. According to the present data, this question can be answered in the affirmative. Giving an arbitrary label along with a nonsense picture has no positive effect on free recall; in fact, it is no better than giving no label at all. The essential equivalence of free recall by subjects given no labels or nonmeaningful ones may reflect the fact that the subjects given no labels constructed their own (as some of their comments suggest), and these subjective labels were about as effective as the arbitrarily assigned ones. In comparison, the advantage of subjects given meaningful labels seems to reflect a true effect of meaningfulness on picture recall.

On the other hand, some benefit can apparently be derived from having even a meaningless label at the time a nonsense picture is presented, if that label is also given at the time the picture is recalled. The role of a meaningless label in the current study seems similar to that of a weak associate in free recall of words (cf. Thomson \& Tulving, 1970). Such a label is not sufficient to aid uncued recall; but when the picture is encoded in the specific context of the meaningless label, its recall is facilitated in the presence of that label. Thus, encoding specificity (Tulving \& Thomson, 1973) seems to apply to recall of both words and pictures.

The results of this study also shed light on the locus of the meaningful-label effect. Specifically, when a meaningful label is given at the time of test only, it fails to facilitate recall. It therefore appears that the label cannot be having its effect solely upon the retrieval process. In fact, if subjects who were given no label at presentation made up their own, the labels given at the time of test may have interfered with retrieval, leading to the poor performance observed. In contrast, when a meaningful label is given only at the time of presentation, recall is better than if no label is given. Thus, labeling does seem to take effect at the time of encoding. And, consistent with the principle of encoding specificity, recall is best when the label is given at the time the picture is encoded and reinstated at the time of retrieval.

In summary, our data suggest that the effect of applying a meaningful label to an otherwise uninterpretable picture is, as Bower et al. (1975) propose, to enable that picture to be encoded as part of a larger schema of cognitive knowledge. This schema is relatively retrievable during free recall and will thus facilitate reproduction of the picture. In contrast, the effect of applying a nonmeaningful label seems to be at a more specific level of picture-label association, one which will facilitate recall given the label as a cue, but will not do so without such a cue.

The similar effects of labeling on picture- and verbal. item recall point to a commonality between the pictorial and verbal domains. They suggest that in both cases, what is stored in memory is an interpretation of what is presented, rather than something like a template of the presented items themselves.

\section{REFERENCE NOTE}

1. Wiseman, G., \& Neisser, U. Perceptual organization as a determinant of visual recognition memory. Paper presented at meeting of the Eastern Psychological Association, 1971.

\section{REFERENCES}

Bower, G. H., Karlin, M. B., \& Dueck, A. Comprehension 
and memory for pictures. Memory \& Cognition, 1975, 3, 216-220.

Bransford, J. D., \& Johrson, M. K. Contextual prerequisites for understanding: Some investigations of comprehension and recall. Journal of Verbal Learning and Verbal Behavior. 1972, 11, 717-726.

Dooling, D. J., \& Lachiman, R. Effects of comprehension on retention of prose. Journal of Experimental Psychology, 1971. 88, 216-222.

Freedman, J., \& Haber, R. N. One reason why we rarely forget a face. Bulletin of the Psychonomic Society, 1974, 3, 107.109.

Nicosia, G. L., \& Santa, J. L. Words and reproduction of nonsense things. Journal of Experimental Psychology, 1975, 104, 369-375.

Price, R. Droodles. Los Angeles: Price/Stern/Sloan, 1972.
Price, R. Droodles \# 2. Los Angeles: Price/Stern/Sloan, 1973.

SANTA, J. L., \& RANKEN, H. B. Effects of verbal coding on recognition memory. Journal of Experimental Psychology, 1972, 93, 268.278.

Thomson, D. M., \& Tulving, E. Associative encoding and retrieval: Weak and strong cues. Joumal of Experimental Psychology, 1970, 86, 255-262.

Tulving, E., \& Thоmson, D. M. Encoding specificity and retrieval processes in episodic memory. Psychological Review, 1973, 80, 352-373.

(Received for publication March 11, 1976; revision accepted April 20, 1976.) 